\title{
How Transcranial Doppler can assess the effect of hyperosmolar therapy and the degree of circulatory compromise in acute brain herniation
}

\author{
Hosam Al-Jehani ${ }^{1,2,3^{*}}$, Mohammad Alkutbi ${ }^{1}$, Mohammad Maleki ${ }^{1}$, Judith Marcoux ${ }^{1}$ and Jeanne Teitelbaum ${ }^{1,2}$
}

\begin{abstract}
Patients in acute neurological extremes secondary to refractory intracranial hypertension are challenging because of the complex management options available to them, especially when compounded with signs of brainstem compromise. Objective evidence of cerebral circulatory compromise is often lacking. We present a case in which an objective evaluation of a cerebral circulatory compromise was documented using transcranial Doppler as well as its resolution with hyperosmolar therapy.
\end{abstract}

Keywords: Transcranial doppler; Cerebral circulatory arrest

\section{Background}

Mannitol is a widely accepted in therapy for the treatment of intracranial hypertension (ICHT), as it has positive effect on cerebral perfusion pressure (CPP) and cerebral blood flow (CBF) more so in those patients with focal injury (vs. diffuse), those with lesions on the brain imaging, and those in whom the CPP is at or below the auto regulation threshold (Brown et al. 1979; Mendelow et al. 1985; Rosner \& Coley 1987; Bratton et al. 2007; Wakai et al. 2007). The mannitol doses reported in these studies ranged from 0.25 to $0.5 \mathrm{gm} / \mathrm{kg}$ bolus of $20 \%$ mannitol given intravenously over 10 to 15 minutes. In acute herniation, high doses $(1.5 \mathrm{~g} / \mathrm{kg})$ have been shown to improve patient outcome (Cruz et al. 2001; Cruz et al. 2002; Cruz et al. 2004).

Transcranial Doppler (TCD) has been widely used for the assessment of cerebral blood flow in several clinical conditions (Topcuoglu 2012) including intracranial hypertension. TCD is gaining acceptance as a rapid, portable and reliable method for assessing intracranial hypertension and cerebral blood flow compromise. In their recent study, Tazarourte et al., reported that $50 \%$ of TCD's performed prior to the arrival to the trauma center were abnormal,

\footnotetext{
* Correspondence: hosam.aljehani@gmail.com

'Department of Neurology and Neurosurgery, Montreal Neurological Institute and Hospital, 3801 University St., Suite 109, Montreal, QC H3A 2B4, Canada

${ }^{2}$ Neurocritical Care Unit, Montreal Neurological Institute and Hospital, Montreal, QC, Canada

Full list of author information is available at the end of the article
}

resulting in administration of hyperosmolar therapy in the ambulance (Tazarourte et al. 2011). Moreover, only patients with abnormal TCD examination required emergency surgery and interestingly those patients in whom the TCD parameter where not improved after the administration of such therapy died within 48 hours (Tazarourte et al. 2011). The case presented in this article illustrates the usefulness of TCD in the monitoring of ICHT leading to herniation and its ability to assess response to therapy.

\section{Case report}

A 49-year-old right-handed male presented to a local community hospital with headache and progressive rightsided weakness. His past medical history included renal cell carcinoma, in remission for the past 3 years, treated by surgical resection and chemotherapy. The computerized tomography $(\mathrm{CT})$ scan of the head performed on the day of presentation revealed a large hemorrhagic lesion in the left central region of the brain highly suspicious of a tumoral bleed, as well as significant peri-lesional edema, severe midline shift and uncal herniation (Figure 1).

Within 2 hours of his arrival, the patient deteriorated, with a drop in Glasgow Coma Scale (GCS) from 15 to 12 prompting urgent intubation prior to transfer to our hospital. Upon arrival in our emergency room, the patient's neurological examination revealed non-reactive pupils, absent corneal reflexes and extremely abnormal eye movements on oculo-cephalic reflex testing. The 


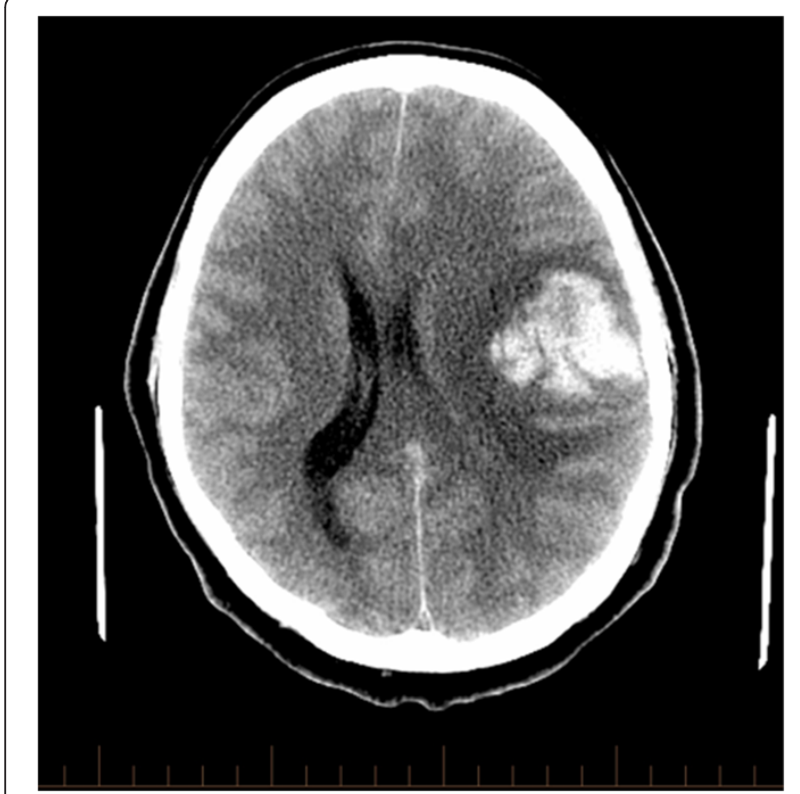

Figure 1 Non-contrast axial CT scan showing large frontal hemorrhage with peri-lesional edema and evidence of mass effect. patient was hyperventilated and given a $200 \mathrm{~mL}$ bolus of $20 \%$ mannitol $(0.58 \mathrm{~g} / \mathrm{kg})$. A repeat CT scan to rule out further hemorrhage showed no change from the previous in terms of hemorrhage, swelling or brain stem compression. A neurologic exam performed immediately after the CT scan, 30 minutes after the mannitol bolus, the patient had deteriorated with absent pupillary response, absent corneal reflexes and a total lack of eye movement on oculocephalic reflex testing, no spontaneous breathing and extensor posturing in the extremities to painful stimuli. It was felt that the patient was not salvageable. Before calling in the family and withdrawing active care, a transcranial Doppler (TCD) was performed in the emergency department to assess cerebral flow. The TCD was performed by the first author (a neurosurgeon and a neuro-intensivest), using a P4-1c Phased Array probe (ZONARE Medical Systems, Inc., Mountain View, CA, USA), operated at $2-3 \mathrm{mHz}$ to insonate the temporal windows bilaterally. There was reverberating flow in the left middle cerebral artery (MCA) compatible with cerebral circulatory arrest on that side, but a high resistance pattern of flow in the right MCA (Bellner et al. 2004). Optic nerve ultrasonography was also performed and showed bilateral dilatation of the optic

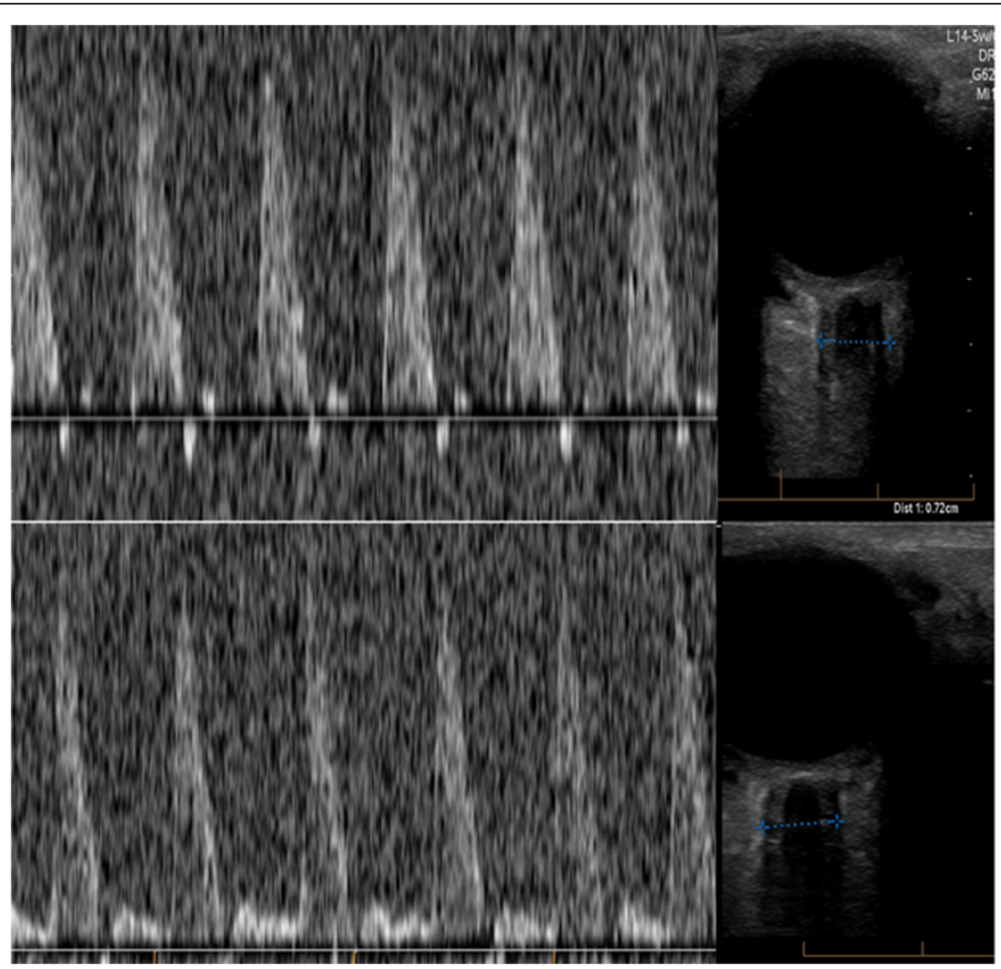

Figure 2 The upper panel shows the transcranial Doppler of the left middle cerebral artery with a prominent systolic spike with a diastolic descent in the Doppler tracing suggestive of reverberating flow seen in circulatory arrest. The lower panel shows the

transcranial Doppler of the right middle cerebral artery with high resistance circulatory pattern with a high systolic peak and low diastolic velocity suggestive of malignant intracranial hypertension, with a pulsatility index ( $\mathrm{PI}=$ peak systolic-end diastolic velocities/mean flow velocity) of 2.5. The optic nerve ultrasonography seen on the right side of the figure are corresponding to the side of the middle cerebral artery insonation and were measuring $7.2 \mathrm{~mm}$ and $6.4 \mathrm{~mm}$ in the left and right optic nerve sheaths, respectively. 
nerve sheath compatible with intracranial hypertension (Soldatos et al. 2009) (Figure 2).

Because of the presence of flow demonstrated in the right MCA, the patient was given another $500 \mathrm{~mL}$ of $20 \%$ mannitol $(1.53 \mathrm{~g} / \mathrm{kg})$ over five minutes with close monitoring of his blood pressure. Within a few minutes the TCD examination showed the return of circulation in both hemispheres along with a reduction in the diameter of the optic nerve sheaths bilaterally (Figure 3). Immediately after the TCD the patient was examined and had reactive pupils. Several minutes later he was localizing to pain with his left side. Based on this favorable response, the patient was rushed to the operating room for a decompressive craniectomy and expansive duraplasty along with evacuation of the hematoma and tissue sampling of the hemorrhagic mass. The patient was observed in the intensive care unit for a few days and then transferred to the neurosurgical ward awaiting further treatment for his lesion (biopsy revealed a glioblastoma). Upon transfer to a rehabilitation center, he still had significant right-sided weakness and dysphasia. He had no residual brain stem dysfunction and repeat imaging showed resolving hemorrhage and residual tumor as expected, as well as a left posterior cerebral artery ischemic stroke likely from the herniation syndrome the patient sustained at the beginning of his hospital course.

\section{Discussion}

This case shows the potential utility of TCD examination in acute neurological deterioration to assess the extent of cerebral circulation compromise, the potential for reversal of circulatory arrest and the efficacy of the chosen dose of hyperosmolar therapy. In this case, the patient had no signs of brain stem function. This would have placed him on a conservative palliative approach given the poor neurological prognosis associated with such condition. The TCD on the other hand showed severely compromised yet persistent cerebral flow in a patient who was otherwise considered unsalvageable. This finding promted the use of an additional large dose of mannitol to objectively assess any changes in the cerebral blood flow dynamics. The TCD done after the mannitol dose demonstrated a tangible effect of hyperosmolar therapy on the cerebral circulation that was later responsible for a reversal of the herniation and the return of brain function. The patient care shifted from a consideration of a palliative approach to aggressive

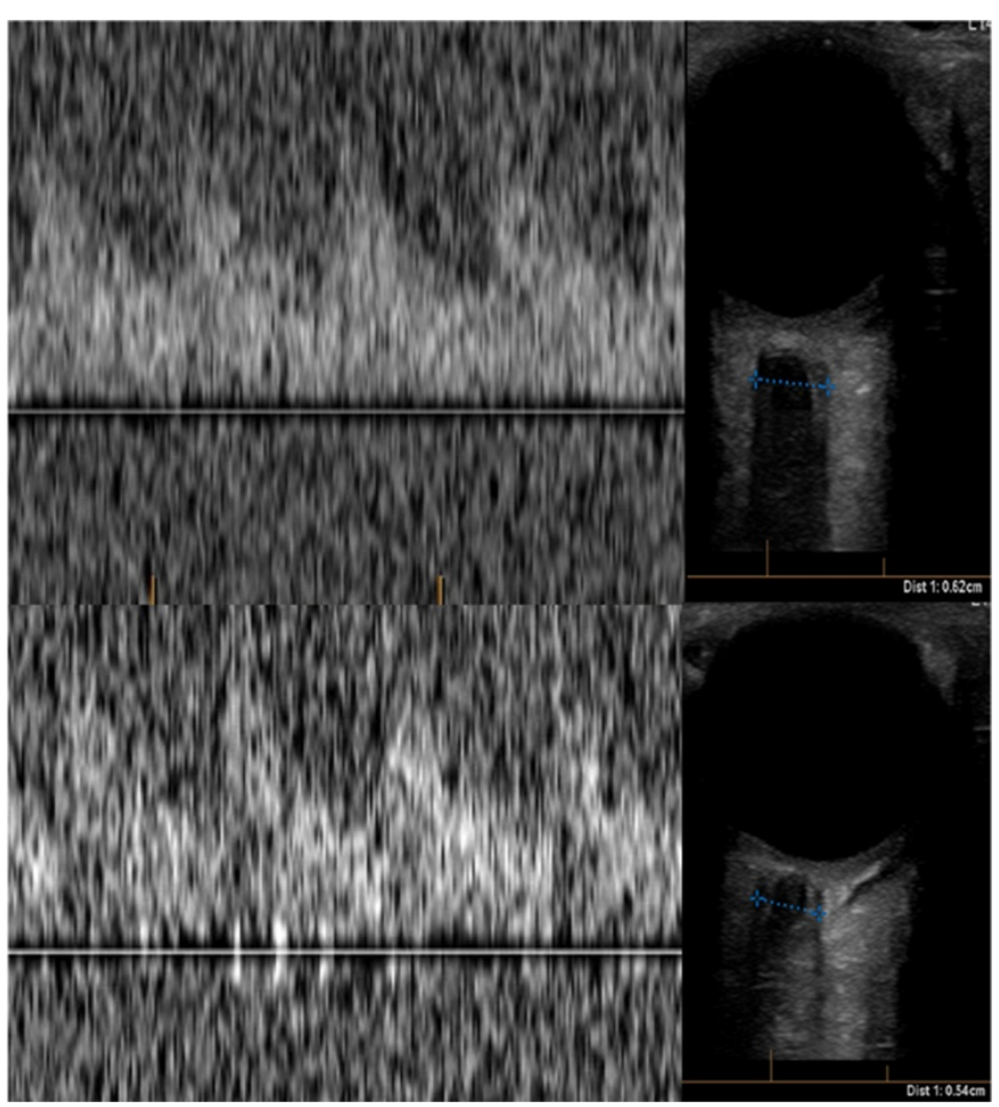

Figure 3 This is the post mannitol and hyperventilation transcranial Doppler showing normal tracing in both middle cerebral arteries (Left upper and lower panels) with PI of $\mathbf{2 . 9}$ bilaterally. In addition, there was a reduction of the diameter of the optic nerve sheath with the left and right side measuring $6.2 \mathrm{~mm}$ and $5.4 \mathrm{~mm}$, respectively. 
surgical and medical therapy with good outcome of the acute care of this patient, not achievable otherwise, had the TCD examination not been performed. This technique may also be useful in gauging whether the chosen dose of hyperosmotic agent is actually the appropriate one. In a pilot study by Tazarourte et al., TCD examination was carried out in a pre-hospital setting, or upon arrival, and patients were treated according to the TCD results of improved blood flow (Tazarourte et al. 2011). TCD was used to assess the efficacy of the maneuvers, and those patients for whom the cerebral perfusion could be corrected according to the pulsatility index fared better (Bellner et al. 2004).

Both TCD and optic nerve ultrasonography can be done with portable machines in the emergency department, intensive care unit and even in the operating room if necessary (Soldatos et al. 2009; Raboel et al. 2012). Performance of TCD examination by a trained technician or a physician is not time consuming. Most TCD units are easily transported to the point of care where the patient is (e.g. The emergency department or the operating room) and newer models even offer portable hand held forms of the device. The learning curve is not discouragingly steep. The major limitation is the logistic constrains of having a trained TCD performer available to capture such patients at these extreme conditions. In conclusion, the use of TCD and optic nerve ultrasonography at the point of care on patients with acute neurological deterioration could be a useful and objective adjunct to help gauge ICP and guide therapy outside of the Intensive Care environment and without the potential delays associated with the installation of invasive monitoring techniques.

\section{Conclusion}

The use of transcranial Doppler in patients presenting with acute neurological deterioration could be a valuable tool to objectively and non-invasively assesses the intracranial pressure dynamics and guide effective course of treatment.

\section{Consent}

Written informed consent was obtained from the patient for the publication of this report and any accompanying images.

\section{Competing interests}

There is no competing interest for the material presented from any of the authors.

\section{Authors' contributions}

HA-J Clinical care and writing of the manuscript; MA Participated in the preparation of the Manuscript; MM Clinical care and review of the manuscript; JM Clinical care and review of the manuscript; JT Clinical care and review of the manuscript. All authors read and approved the final manuscript.

\section{Author details}

'Department of Neurology and Neurosurgery, Montreal Neurological Institute and Hospital, 3801 University St., Suite 109, Montreal, QC H3A 2B4,
Canada. ${ }^{2}$ Neurocritical Care Unit, Montreal Neurological Institute and Hospital, Montreal, QC, Canada. ${ }^{3}$ Department of Neurosurgery, King Fahad University Hospital, Dammam University, Al-Khobar, Saudi Arabia.

Received: 14 May 2013 Accepted: 9 July 2013

Published: 15 July 2013

\section{References}

Bellner J, et al. (2004) Transcranial Doppler sonography pulsatility index (PI) reflects intracranial pressure (ICP). Surg Neurol 62(1):45-51. discussion 51

Bratton SL, et al. (2007) Guidelines for the management of severe traumatic brain injury. II. Hyperosmolar therapy. J Neurotrauma 24(Suppl 1):S14-S20

Brown FD, et al. (1979) Detailed monitoring of the effects of mannitol following experimental head injury. J Neurosurg 50:423-432

Cruz J, Minoja G, Okuchi K (2001) Improving clinical outcomes from acute subdural hematomas with the emergency preoperative administration of high doses of mannitol: a randomized trial. Neurosurgery 49(4):864-871

Cruz J, Minoja G, Okuchi K (2002) Major clinical and physiological benefits of early high doses of mannitol for intraparenchymal temporal lobe hemorrhages with abnormal pupillary widening: a randomized trial. Neurosurgery 51(3):628-637. discussion 637-8

Cruz J, et al. (2004) Successful use of the new high-dose mannitol treatment in patients with Glasgow Coma Scale scores of 3 and bilateral abnormal pupillary widening: a randomized trial. J Neurosurg 100(3):376-383

Mendelow AD, et al. (1985) Effect of mannitol on cerebral blood flow and cerebral perfusion pressure in human head injury. J Neurosurg 63:43-48

Raboel PH, et al. (2012) Intracranial pressure monitoring: invasive versus Noninvasive methods-a review. Crit Care Res Pract 2012:14

Rosner MJ, Coley I (1987) Cerebral perfusion pressure: a hemodynamic mechanism of mannitol and the post-mannitol hemogram. Neurosurgery 21:147-156

Soldatos T, et al. (2009) Optic nerve sonography: a new window for the noninvasive evaluation of intracranial pressure in brain injury. Emerg Med J 26 (9):630-634

Tazarourte K, et al. (2011) Pre-hospital transcranial Doppler in severe traumatic brain injury: a pilot study. Acta Anaesthesiol Scand 55(4):422-428

Topcuoglu MA (2012) Transcranial Doppler ultrasound in neurovascular diseases: diagnostic and therapeutic aspects. J Neurochem 123(Suppl 2):39-51

Wakai A, Roberts I, Schierhout G (2007) Mannitol for acute traumatic brain injury. Cochrane Database Syst Rev(1):CD001049

doi:10.1186/2193-1801-2-319

Cite this article as: Al-Jehani et al:: How Transcranial Doppler can assess the effect of hyperosmolar therapy and the degree of circulatory compromise in acute brain herniation. SpringerPlus 2013 2:319.

\section{Submit your manuscript to a SpringerOpen ${ }^{\odot}$ journal and benefit from:}

- Convenient online submission

Rigorous peer review

- Immediate publication on acceptance

- Open access: articles freely available online

- High visibility within the field

- Retaining the copyright to your article

Submit your next manuscript at springeropen.com 